\title{
SOCIAL NETWORK ANALYSIS: UM ENSAIO SOBRE A APLICABILIDADE NA ANÁLISE DE COOPERAÇÃO EM CONTEXTOS ESPORTIVOS
}

Filipe Manuel Clemente, Escola Superior de Educação de Coimbra - ESEC, Coimbra Portugal

Fernando Manuel Lourenço Martins, Escola Superior de Educação de Coimbra- ESEC, Coimbra-Portugal

Rui Sousa Mendes, Escola Superior de Educação de Coimbra-ESEC, Coimbra-Portugal

\section{RESUMO}

A cooperação entre companheiros de equipa e a forma como a mesma decorre é de fundamental importância para a compreensão dos processos sociais no interior de um grupo de trabalho no esporte. Para mensurar esta cooperação, métricas recentes baseadas na teoria de grafos tem fornecido uma nova abordagem capaz de potenciar as informações recolhidas quantitativamente. Assim, o presente ensaio tem como objetivo dar a conhecer as técnicas e possibilidades do Social Network Analysis no esporte e como tal abordagem poderá potenciar os estudos sociais. Análise das propriedades da rede de cooperação, bem como, métricas de determinação de dependência entre companheiros e centralidade individual serão apresentados e discutidos neste ensaio.

Palavras-Chave: Sociometria; Teoria de Grafos; Cooperação; Network.

\section{SOCIAL NETWORK ANALYSIS: AN OVERVIEW ABOUT THE APPLICATION ON COOPERATION ANALYSIS IN SPORTS CONTEXTS}

\begin{abstract}
The cooperation between teammates and the way in which such cooperation occurs is fundamental to understand the social processes within a team. In the particular case of sport such cooperation is inevitable in team sports. Therefore, recent metrics based in Graph Theory have been providing a new approach that allows potentiating the understanding of quantitative information. Therefore, this review aims to share the techniques and possibilities of Social Network Analysis in the specific case of sport and how such approach can potentiate the social studies. Will be presented and discussed in this review techniques to analyze the network properties, as well as metrics to determine the dependency between teammates and the prominence levels of players.
\end{abstract}

Key-Words: Sociometry; Graph Theory; Cooperation; Network. 


\title{
SOCIAL NETWORK ANALYSIS: UNA VISIÓN GENERAL SOBRE LA APLICACIÓN EN EL ANÁLISIS DE LA COOPERACIÓN EN LOS CONTEXTOS DEPORTIVOS
}

\begin{abstract}
RESUMEN
La cooperación entre los compañeros de equipo y la forma en que se ejecuta es de fundamental importancia para la comprensión de los procesos sociales dentro de un grupo de trabajo. En el caso particular del deporte, la cooperación es inevitable en los deportes colectivos. Métricas recientes basados en la teoría de grafos proporcionan un nuevo enfoque capaz de aprovechar la información recopilada cuantitativamente. Por lo tanto, este ensayo tiene como objetivo dar a conocer las posibilidades y técnicas de análisis de redes sociales en el caso específico del deporte y como tal enfoque puede mejorar los estudios sociales. Análisis de las propiedades de la red de cooperación, así como métricas para determinar la dependencia entre los compañeros y la centralidad de lo individuo se presentan y discuten en este trabajo.
\end{abstract}

Palabras-Clave: Sociometría; Teoría de Grafos; Cooperación; Red. 


\section{INTRODUÇÃO}

A interação entre companheiros no interior de uma equipa desportiva é inevitável e fundamental, onde aspetos como coesão e hierarquia entre jogadores encontra-se sempre presente. ${ }^{1-3}$ Pela sua necessidade e essência estes tópicos são regularmente investigados, sempre com elevado grau de complexidade e desafio. ${ }^{4-5}$ Apesar de algumas técnicas de observação e metodologias de avaliação, o facto é que o processo de avaliação da interação, cooperação e coordenação de companheiros de equipa revela quatro principais problemas $^{1}$ : i) a escassa validação instrumental; ii) reduzidos tamanhos das amostras; iii) problemas relacionados com o pressuposto de independência dos membros da equipa; iv) delineação do individual e grupo.

Nas relações no interior do grupo a própria hierarquia pode não ser sinónimo de assertividade no processo observacional dos investigadores. Considere-se o caso do capitão de equipa. Neste caso particular, a sua posição no seio do grupo é claramente identificada como hierarquicamente mais elevada pelo que, pressupostamente, a sua condição de poder e soberania poderia ser avaliada através da nomeação dos seus pares. ${ }^{6}$ No entanto, este poder e hierarquia poderá ser meramente entendida a um nível formal, pelo que, em casos concretos o relacionamento e interação de forma informal poderão revelar outros tipos de direcionamento das conexões interpessoais. ${ }^{7}$ De facto, num estudo bastante interessante realizando em Flandres (Bélgica), o mito do capitão de equipa como principal líder foi desmontado. $^{8}$

Para tal, os autores ${ }^{8}$ classificaram e definiram quatro tipos de líder: i) task leader (líder qua ajuda a equipa a focar-se em campo e atingir os objectivos, intervindo ao nível tático e tomada de decisão); ii) motivational leader (o maior motivador em campo, encorajando sempre os companheiros a fazer melhor); iii) social leader (líder que assume papéis para além do campo, promovendo boas relações entre companheiros e promovendo um bom ambiente de equipa); e iv) external leader (assume-se como o link entre a equipa e pessoas externas ou entidades). Definidos os tipos de líderes, os participantes do estudo identificaram os companheiros que mais se ajustavam a cada tipo de liderança. Os resultados revelaram que apenas $1 \%$ dos participantes identificaram o seu capitão como o melhor líder os quatro papéis possíveis. Adicionalmente, 43,6\% dos participantes 
descreveram que o capitão de equipa não é o melhor líder em um dos quatro domínios possíveis.

Este interessante exemplo é mostra que a análise do comportamento de interação e como os líderes e hierarquias são definidos nem sempre são lineares quanto às suas conclusões. Um dos factores poderá associar-se às metodologias utilizadas, bem como, à pressão social a que os participantes estão sujeitos. De facto, a é expectável que existe interdependência e, consequentemente, liberdade total na resposta por parte dos participantes. ${ }^{1}$ No entanto, nem sempre esta situação é evidente principalmente em relações entre mais jovens e adultos, onde o fator social (no interior da equipa, entendendo-a como uma micro sociedade) determina comportamentos de dependentes dos mais jovens para os mais velhos ${ }^{9}$. Assim, as hierarquias sempre constranger as nomeações e as relações.

Para avaliar e controlar os processos de relacionamento anteriormente aflorados, procurando reduzir o carácter subjetivo, alguns métodos quantitativos têm vindo a ser adotados, emergindo assim a sociometria. ${ }^{10}$ Resultante da sociometria novas abordagens têm vindo a ser desenvolvidas mais recentemente procurando entender a rede de interrelações numa equipa. É desta forma que emerge a análise social de rede (Social Network Analysis - SNA) que tendo como bases a sociometria e a teoria de grafos, aplicando técnicas matemáticas para determinar as relações num entendimento de rede de conexões. $^{11}$

No sentido de descrever as possibilidades da SNA para a compreensão das relações entre companheiros de equipa, o objetivo do presente artigo é providenciar um ensaio que analise as principais definições e conceitos desta abordagem, bem como, disponibilizar um conjunto de métricas originárias da teoria de grafos que permitem identificar matematicamente no interior da rede as características e propriedades da mesma, bem como, identificar os níveis de centralidade dos membros e as suas dependências entre pares. 


\section{SOCIAL NETWORK ANALYSIS: DEFINIÇÕES E CONCEITOS GERAIS}

O SNA é um procedimento matemático que analisa a cooperação de membros constituintes através de técnicas e métricas baseados na teoria de grafos. ${ }^{12}$ A teoria de grafos estuda os grafos numa abordagem matemática utilizando técnicas para modelar relações emparelhadas entre vértices (nós). Algumas definições essenciais associadas à teoria são: ${ }^{13}$ i) grafos direcionados; ii) grafos não direcionados; e iii) grafos ponderados.

O grafo direcionado pode ser definido como uma ordem tripla $G=(V, E, f)$ onde $f$ é a função que mapeia cada elemento em $E$ para o par ordenado de vértices $V$, assim os pares ordenados de vértices são designados de vértices, arcos ou setas direcionadas. ${ }^{13}$ Neste caso $E=(i, j)$ é considerado como tendo direção de $i$ para $j$. O grafo não direcionado pode ser entendido como grafo $G$ que é definido pelo par $(V, E)$ onde $V$ é o conjunto de vértices representado os nós e $E$ é um conjunto de arestas representando a conexão entre nós. Um caso de grafo não direcionado é $E=\{(i, j) \mid i, j \in V\}$ com uma única conexão entre os vértices $i$ e $j$ que são vizinhos. ${ }^{13}$ Por fim, um grafo ponderado, $A_{i, j}$ os valores assumem um número real entre 0 e $1 . A_{i, j}$ especifica a força de conexão entre os vértices $i$ e $j .^{14}$

A teoria de grafos e a análise de rede têm sido utilizadas em diversas áreas do conhecimento, desde no estudo de processos biológicos, ${ }^{15}$ à interação social ${ }^{16}$ ou aos sistemas de informação. ${ }^{17}$ No caso dos estudos no plano social, diversas aplicações podem ser verificadas, desde à gestão, ${ }^{18}$ antropologia ${ }^{19}$ ou sociologia. ${ }^{20}$

Outra área onde a teoria de grafos e o SNA pode ser evidentemente aplicada é ao desporto, particularmente no estudo das relações interpessoais em desportos coletivos. ${ }^{21}$ Neste caso o SNA pode ser definido como uma análise estrutural. ${ }^{22}$ O SNA não é uma teoria formal, é antes um estratégia de investigação das estruturas sociais. ${ }^{23}$ A maior preocupação do SNA é analisar a relação entre atores, mais do que a normal preocupação dos estudos de focar a análise eminentemente no indivíduo, independentemente do grupo. ${ }^{24}$ Assim, a análise da rede preocupa-se com a estrutura e padrão de relacionamento entre atores, procurando identificar as suas causas e consequências. ${ }^{25}$ 
Uma rede social pode ser considerada como um conjunto finito de atores e relações entre eles. ${ }^{26}$ Desta forma, os dados da rede são definidos como atores (vértices ou nós) e relações (arestas). ${ }^{27}$ Geralmente, a análise da rede foca-se nas relações entre vértices, assim o estudo centra-se na relação global entre os membros do grupo e não num vértice em específico. ${ }^{28}$ Alguns conceitos no SNA podem ser determinantes para a sua compreensão, tais como: ${ }^{26}$ i) díade, sendo o nível mais baixo de interação que representa uma ligação entre dois vértices; ii) tríade, que representa uma relação triangular e as possíveis ligações entre os membros; iii) subgrupo, que representa um qualquer conjunto de vértices e as possibilidades de ligação entre eles; iv) grupo, que representa o sistema inteiro de vértices e as possibilidades de conexões entre eles.

Todos estes conceitos podem ser encontrados em desportos coletivos ou em contextos da Educação Física pelas suas características específicas de interação entre pares que resultam das propriedades próprias dos contextos e regras. ${ }^{29}$ De facto, as aplicações no campo das Ciências do Desporto podem ser amplas e diversificadas. No entanto, as de maior pertinência evidente poderão associar-se com a percepção de como os companheiros de equipa se relacionam no interior do campo e fora do contexto de treino. Esta percepção de interação social poderá ser determinante para identificar como a equipe se envolve em torno de um objetivo comum, ou que jogadores são fundamentais para promover a conectividade entre companheiros. Outra possibilidade de aplicação será na Educação Física, analisando como os estudantes se conectam e avaliar se a conexão global é uniformemente distribuída ou se existem casos de grupos no interior da rede geral da turma.

Como aflorado anteriormente, as possibilidades de aplicação do SNA no campo das Ciências do Desporto e Educação Física são diversificadas e, como tal, as métricas existentes serão apresentadas de seguida em três grandes grupos de análise: i) análise geral da rede; ii) análise da relação de dependência entre membros da rede; e iii) análise da centralidade dos membros da rede. 


\section{PRÉ-PROCESSAMENTO DOS DADOS: DESENVOLVIMENTO DA MATRIZ DE ADJACÊNCIA}

Para que o grafo surja é necessário obter uma matriz de adjacência que permita identificar o direcionamento das interações entre membros constituintes da rede. A matriz de adjacência é uma tabela de dupla entrada em que, no caso do network, os valores correspondem ao nível de interação entre vértices. Consideremos o seguinte caso: uma equipa de futsal constituída por 5 membros foi analisada quanto ao nível de classificação do melhor amigo. Cada membro poderia votar apenas uma vez num colega. Neste caso, 1 será o valor de conexão e 0 o valor de não conexão. Apresenta-se de seguida a matriz de adjacência e o grafo correspondente (FIGURA 1).

\begin{tabular}{cccccc} 
& J1 & J2 & J3 & J4 & J5 \\
J1 & 0 & 0 & 0 & 0 & 1 \\
J2 & 0 & 0 & 0 & 0 & 1 \\
J3 & 0 & 1 & 0 & 0 & 0 \\
J4 & 0 & 0 & 1 & 0 & 0 \\
J5 & 0 & 1 & 0 & 0 & 0 \\
\hline
\end{tabular}
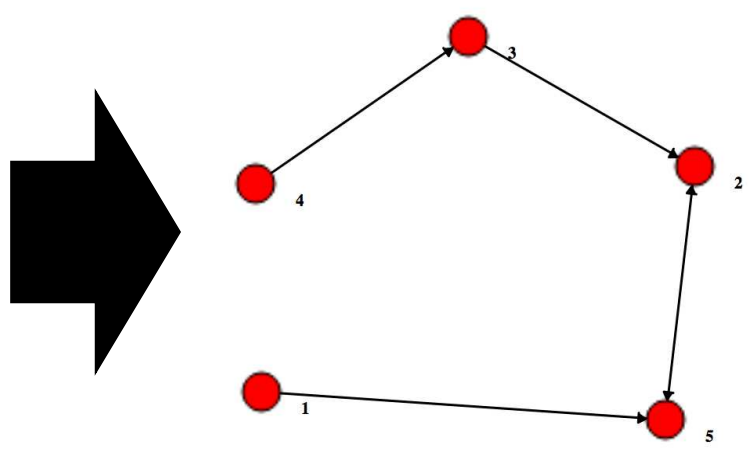

Figura 1: Exemplo de matriz de adjacência e respetivo grafo.

Como é possível verificar na matriz de adjacência (FIGURA 1) os jogadores 2 e 5 são os que recebem mais indicações dos seus companheiros (2 nomeações cada), refletindo-se tais resultados no grafo correspondente. Por outro lado, os jogadores 1 e 4 não receberam qualquer votação por parte dos companheiros de equipa, verificando-se no grafo o seu isolamento, apresentando apenas grafos direcionados para os elementos em quem votaram.

Este tipo de exemplo poderia ser realizado em qualquer outro tipo de análise de interações sendo que o valor 1 para conexão pode variar em casos de maiores ponderações. Casos como escalas de likert em que podem atribuir de 0 (não amigo) até 5 (melhor amigo), passando por classificações intermédias (e.g., pouco amigo, amigo), são exemplos de matrizes de adjacência com ponderações.

Importa considerar que por si só estas matrizes e grafos correspondentes, apesar de permitirem uma visualização inicial do estado de conectividade da rede, não permitem um Conexões: revista da Faculdade de Educação Física da UNICAMP, Campinas, v. 13, n. 3, p. 175-194, jul./set. 2015. ISSN: 1983-9030 
conhecimento profundo sobre as propriedades e tendências de interação entre membros. Para tal efeito, são necessárias métricas que classificam e caracterizam determinados parâmetros pelo que, de seguida, serão apresentadas e descritas tais métricas, bem como, discutidas as suas possibilidades de interpretação.

\section{ANÁLISE GERAL DA REDE DE COOPERAÇÃO: MÉTRICAS POSSÍVEIS DE ANÁLISE}

A análise geral da rede permite identificar as propriedades e característica num ponto de vista meramente global. Possibilita identificar níveis de conexão entre membros, número de conexões totais, densidade das conexões, diâmetro da rede ou coeficientes de agrupamento.

\subsection{Total de Conexões}

Cada elemento $(i, j)$ da matriz de adjacência poderá representar o número de interações do elemento $i$ para o $j$. A soma dos elementos pertences à matriz de adjacência $\sum_{j \neq i}^{n}(i, j)$ será o número de interações do element $i$ para todos os restantes membros da rede. Assim, a soma das linhas $L=\sum_{i}^{n} \sum_{j \neq i}^{n}(i, j)$ permite identificar o total de conexões entre membros da rede. No grafo, este número é o total de linhas entre todos os vértices.

Como interpretação é possível determinar que maiores valores de total de conexões representa a maior cooperação entre os membros da rede. Pode ser igualmente associado com a maior probabilidade de sucesso nas interações entre companheiros, significando uma mais forte organização estrutural da rede.

\subsection{Densidade do Grafo}

$\mathrm{Na}$ teoria de grafos, a densidade de um grafo é a proporção da possibilidade máxima de linhas que estão presentes entre vértices. A densidade de rede mostra como é disperso ou denso o grafo de acordo com o número de conexões por vértice. ${ }^{13}$ Devido ao facto de um grafo ser constituído por um número finito de vértices (designados de $n$ ), no caso de grafos não direcionados eles podem ter o máximo $n(n-1) / 2$ possível de pares entre vértices e $n(n-1) / 2$ possível de arestas (dividido por 2 porque a conexão $(i, j)$ é a mesma que $(j, i)$ não sendo necessário contá-los duas vezes). A densidade $\Delta$ do grafo é definido como o rácio do 
total de conexões presents em $L$ para o máximo possível de número de conexões que podem existir:

$$
\Delta=\frac{L}{n(n-1) / 2} \text { ou } \Delta=\frac{2 L}{n(n-1)} \text {. }
$$

No caso de relações ordenadas, como em escalas de likert, a possibilidade de conexões diretas num diágrafo de $n$ vértices é $n(n-1)$, assim a densidade é calculada como:

$$
\Delta=\frac{L}{n(n-1)} \text {. }
$$

Em ambos os casos a densidade é a fração tendo como mínimo 0 (sem linhas) e máximo 1 (todas as linhas). Considerando que a densidade avalia a afetação geral entre membros da rede, é possível interpretar valores próximos de 1 como todos os membros fortemente ligados entre si e valores inferiores a 0,5 como a presença de maior ambiguidade no relacionamento.

\subsection{Distância do Grafo}

$\mathrm{Na}$ teoria de grafos, dois vértices conectam-se se existir uma sequência de vértices e arestas adjacentes de uns para outros. No exemplo 1-3-4-5-3-2-6 o membro 1 encontra-se ligado ao 6 através da sequência de passos (walks). Ainda no mesmo exemplo o caminho mais próximo para chegar ao membro 6 ocorre na sequência 3-2-6. A distância geodésica entre dois vértices define-se como o comprimento geodésico entre eles, assim é possível definir a distância entre vértices $n_{i}$ e $n_{j}$ como $d(i, j) .{ }^{(26)}$ A distância entre dois vértices é o caminho mais curto entre vértices e em casos em que nenhum caminho é formado definese como $d(i, j)=\infty$, assumindo que os vértices estão tão longe entre eles que não se podem conectar ${ }^{13}$.

O comprimento médio do caminho num grafo é definido como o valor médio e máximo do valor de $d(i, j)$ considerando todos os pares de distintos vértices, $i, j \in V(G)$ que está conectado pelo menos um caminho, assim a média do caminho de uma rede é o valor médio the arestas entre membros, que podem ser cruzados no caminho mais curto entre dois vertices: 


$$
d=\frac{2}{N(N-1)} \sum_{i=1}^{N} \sum_{j=1}^{N} d_{\min }(i, j)
$$

onde $d_{\min }(i, j)$ é a distância mínima entre vertices $i$ e $j$.

Como interpretação dos valores obtidos poder-se-á referir que médias mais baixas representam melhor conexão entre companheiros visto estarem mais próximos entre si na rede geral. Valores médios superiores sugerem que os membros se encontram mais afastados entre si e, portanto, mais dispersos e desconectados.

\subsection{Diâmetro do Grafo}

O diâmetro $D$ do grafo correspondente relaciona-se com a distância entre vértices. O diâmetro de um grafo é a distância máxima (o comprimento geodésico mais largo) entre qualquer par de vértices e é calculado pela seguinte fórmula: ${ }^{26}$

$$
D=\max _{i, j} d_{\min }(i, j)
$$

O diâmetro tem um mínimo de 1 , quando todos os vértices estão diretamente conectados entre eles e um máximo de $n-1$ quando o caminho entre um par de vertices passa entre um qualquer outro vértice da rede. Assim, o diâmetro do grafo é uma importante métrica dado que reflete quão longe estão dois vertices no grafo. No caso da análise em contexto desportivo, poderá permitir averiguar como a rede de amizade se estreita no conjunto da equipa ou da turma de educação física.

\subsection{Coeficiente de Agrupamento}

$\mathrm{O}$ coeficiente de agrupamento quantifica quão junto um vértice e o seu vizinho se encontram num grafo ou sub grafo. ${ }^{30}$ No caso de grafos diretos o coeficiente de agrupamento de um vértice é a proporção de conexões presentes entre vértices diretamente conectados (designando-os de vizinhos $N$ daquele vértice). Assim, o coeficiente de agrupamento de cada vértice $i$ é calculado como a fração do número de todos os $\operatorname{arcos} a_{j k}$ entre o vértice $k_{i}$ e seus vizinhos dividido pelo número máximo $k_{i}\left(k_{i}-1\right)$ de conexões que poderiam existir entre eles: 


$$
C_{i}=\frac{\left|\left\{a_{j k}, a_{j k} \in E\right\}\right|}{k_{i}\left(k_{i}-1\right)}
$$

para todos vértices $j, k$ na vizinhança $N$ de $i$.

Desta forma, o coeficiente de agrupamento quantifica o grau de interconetividade na vizinhança de um vértice. Quanto maior ele é (valor), mais perto o vértice e seus vizinhos estão.

Uma rede com elevado nível de coeficiente de agrupamento significa que os seus vértices tendem a formar facções, podendo sugerir falta de cooperação global e baixa interconectividade com membros a comunicar mais com determinados vértices e não de forma geral.

\section{DEPENDENCIA ENTRE MEMBROS DA REDE DE COOPERAÇÃO: MÉTRICAS POSSÍVEIS DE ANÁLISE}

A cooperação entre membros pode ser marcada pela dependência de um membro pelo o outro. Assim a sobreposição topológica ${ }^{14,31}$ é utilizada para representar o par de membros que cooperam com os mesmos companheiros. Se dois membros participam no mesmo evento ou atividade com os mesmos companheiros poder-se-á referir que surge uma relação triangular. No entanto, o jogador $i$ pode ser mais dependente do $j$ se ele apenas participar nas mesmas atividades de $j$. Como resultado deste conceito é possível definir a dependência topológica $T_{d}=\left[t d_{i j}\right] \in \mathbb{R}^{n \times n}$ como: $^{32}$

$$
t d_{i j}= \begin{cases}\frac{\sum_{i \neq i, j} r_{i l} r_{l j} r_{i j}}{\sum_{l \neq i} r_{i l}}, & \text { if } i<j \\ \frac{\sum_{l \neq i, j} r_{i l} r_{l j} r_{i j}}{\sum_{l \neq i} r_{l j}}, & \text { if } i>j \\ 1, & \text { if } i=j\end{cases}
$$

com $i, j, l=1,2, \ldots, n$

Como consequência, dois membros têm uma maior dependência topológica, $t d_{i j}=1$ se eles participarem nas atividades com o mesmo membro e com outro companheiro formando um grupo mais forte. 
No entanto, desde que $T_{d}$ corresponda à matriz quadrada com o tamanho igual ao número de membros e desde que contrariamente à matriz de adjacência ou sobreposição topológica, ${ }^{14} T_{d}$ não é simétrica, assim $t d_{i j} \neq t d_{j i}$, o que dificulta comparar os pares $t d_{i j} \mathrm{e}$ $t d_{j i}{ }^{32}$

Para complementar o conceito prévio, uma métrica nova é gerada designando-se de interdependência $T_{i d}=\left[t i_{i j}\right] \in \mathbb{R}^{n \times n}$ calculada como. ${ }^{32}$

$$
T_{i d}=T_{d}-T_{d}^{t}
$$

onde $T_{d}^{T}$ é a transposição da matriz $T_{d}$ e $T_{d i}$ que corresponde a uma matriz quadrada antissimétrica, $t i_{i j}=-t i_{j i}$. No caso desportivo é possível determinar dependência entre membros se $t i_{i j}>0$, logo o membro $i$ depende do $j$ para realizar a atividade.

\section{ANÁliSE DA CENTRALIDADE E PROEMINÊNCIA DOS OBSERVADOS: MÉTRICAS POSSÍVEIS DE ANÁLISE}

Para além da análise realizada às propriedades da rede, bem como, a identificação de casos de dependência entre pares, outra análise possível e necessária é a caracterização da proeminência de determinado membro na sua rede de cooperação. Para tal, um conjunto de métricas de centralidade podem ser aplicadas identificando o contributo individual de cada membro na equipa, bem como, o nível do seu destaque.

\subsection{Grau de Centralidade}

A centralidade do membro significa que o vértice deve ser o mais ativo no sentido em que é o que possui mais ligações com os seus companheiros na rede. ${ }^{26}$ A medida de centralidade de um membro deve ser o grau do vértice $d\left(n_{i}\right)$, assim é possível definir $C_{D}\left(n_{i}\right)$ como o nível de centralidade do membro:

$$
C_{D}\left(n_{i}\right)=d\left(n_{i}\right)=x_{i+}=\sum_{j} x_{j i}=\sum_{j} x_{j i}
$$

Para além deste algoritmo, outro pode ser utilizado para avaliação padrão: 


$$
C_{(D)}^{\prime}\left(n_{i}\right)=\frac{d\left(n_{i}\right)}{g-1}
$$

que é a proporção de vértices adjacentes a $n_{i}$. Neste caso, $C_{(D)}^{\prime}\left(n_{i}\right)$ é independente de $g$, e pode ser comparado com grafos de diferentes tamanhos. ${ }^{26}$ Nesta análise o membro com maior nível de grau de centralidade pode ser designado de eixo, visto ser o mais conectado com os restantes vizinhos. ${ }^{13}$

A interpretação desta métrica permite identificar que os maiores valores revelam "onde a ação decorre" no grafo. ${ }^{26}$ No fundo, pode ser entendido como o companheiro que recebe mais conexões e se conecta com mais companheiros numa equipa ou numa turma de Educação Física. É, portanto, o centro da equipa no que se refere a determinada análise.

\subsection{Centralidade de Proximidade}

A centralidade de proximidade indica os vértices que podem mais rapidamente interagir com os restantes vértices da rede, pela sua proximidade quanto à distância. ${ }^{13}$ Considerando $d\left(n_{i}, n_{j}\right)$ como o número de linhas numa conexão geodesica dos vértices $i$ e $j$, a distância total de $i$ dos restantes membros é $\sum_{j=1}^{g} d\left(n_{i}, n_{j}\right)$ onde a soma é obtida de todos $j \neq i^{26}$. Neste sentido, a proximidade pode ser calculada como:

$$
C_{(C)}\left(n_{i}\right)=\left[\sum_{j=1}^{g} d\left(n_{i}, n_{j}\right)\right]^{-1}
$$

Outra fórmula possível pode ser utilizada para realizar comparações entre grafos de diferentes tamanhos:

$$
\begin{gathered}
C^{\prime}{ }_{(C)}\left(n_{i}\right)=\frac{g-1}{\left[\sum_{j=1}^{g} d\left(n_{i}, n_{j}\right)\right]} \\
=(g-1) C_{c}\left(n_{i}\right) .
\end{gathered}
$$


No caso particular deste índice, a amplitude oscila entre 0 e 1 e pode ser vista como a média inversa da distância entre o vértice $i$ e os restantes vértices. ${ }^{26}$ De forma geral, valores inferiores de centralidade de proximidade são originados por um aumento na distância entre caminhos para chegar aos vizinhos. ${ }^{13}$

\subsection{Centralidade de Intermediação}

A centralidade de intermediação mede os vértices intermediários entre vizinhos. Sem estes vértices a distância entre vizinhos aumenta pela sua não conexão direta. Nesse sentido, a centralidade de intermediação mostra a importância desses membros que se encontram numa grande proporção do caminho de outros vértices na rede. ${ }^{13}$ Para vértices distintos $i, j, k \in V(G)$, considere-se $g_{i j}$ como sendo o número total de caminhos mais curtos entre $i$ e $j$, e $g_{i j}(k)$ como sendo o número de caminhos mais curtos de $i$ para $j$ passando por $k$. Para além disso, para $k \in V(G)$, considere-se $V(i)$ como o conjunto de todos os pares ordenados, $(i, j)$ em $V(G) \times V(G)$, de tal forma que $i, j, k$ são todos distintos. ${ }^{13}$ Assim, a centralidade de proximidade é calculada como:

$$
C_{b}(k)=\sum_{(i, j) \in V(k)} \frac{g_{i j}(k)}{g_{i j}}
$$

Existirá, portanto, um mínimo de 0 quando $k$ cai na geodésicas. ${ }^{26} \mathrm{O}$ máximo será atingido quando todos os vértices caírem na geodésicas. Sumariamente, esta métrica pode ser utilizada para identificar o membro que mais gera caminhos curtos na equipa, podendo ser o "amigo em comum" que promove a ligação entre membros não conexos.

\subsection{Centralidade de Excentridade}

A centralidade de excentridade mede quão acessível um vértices relativamente a outros. ${ }^{13}$ Assim, considere-se $G=(V, E)$ como um grafo não direcionado. A excentridade pode ser calculada como:

$$
C_{e c c}=\frac{1}{\max \{\operatorname{dist}(i, j)\}}
$$

onde $\operatorname{dist}(i, j)$ é o caminho mais curto entre os vértices $i$ e $j$. 
A excentridade $C_{e c c}$ de um vértice $V$ é a maior distância entre $v$ e qualquer outro vértice. ${ }^{13}$ Como interpretação dos resultados, vértices com elevados valores significa que é fácil estar conectado com a equipa. Por outro lado, valores mais baixos revelam que os vértices estão afastados dos restantes companheiros.

\subsection{Grau de Prestígio}

O grau de prestígio mede o grau de entrada de determinado vértice que pode ser definido como $d_{I}\left(n_{i}\right)$. No caso da análise social em context desportivo, pode ser o membro que recebe mais votações dos companheiros como elemento fundamental na equipa, calculando-se por: ${ }^{26}$

$$
P_{D}\left(n_{i}\right)=d_{I}\left(n_{i}\right)=x_{+i}
$$

De forma a generalizar de acordo com o tamanho do grupo $g$, é possível calcular da seguinte forma:

$$
P_{D}^{\prime}\left(n_{i}\right)=\frac{x_{+i}}{g-1}
$$

Como interpretação, os valores mais elevados representam o vértice com maior prestígio, assim poderá ser o que é mais reconhecido e votado pelos seus companheiros durante a determinação do melhor companheiro de equipa. $\mathrm{O}$ valor mais elevado do grau de prestígio ocorre quando todos os votos são direcionados para o mesmo membro.

\section{CONSIDERAÇÕES FINAIS}

A identificação de padrões de interação num grupo de trabalho possibilita a compreensão sobre processos sociais que podem potenciar o desempenho coletivo. Independentemente do contexto, a capacidade de investigar as conexões entre membros de uma equipa determina a proximidade da compreensão das causas e fatores associados com a evolução relacional do grupo. Assim, a sociometria é inevitavelmente uma possibilidade válida e fidedigna que permite uma abordagem metodológica para a caracterização do relacionamento entre pares. 
No caso específico do desporto, o relacionamento entre companheiros determina a evolução da dinâmica coletiva em termos sociais que, por sua vez, se repercutem no desempenho coletivo. Assim, urge identificar os padrões de interação entre companheiros. Apesar de diferentes opções metodológicas, a quantificação apesenta vantagens irrefutáveis relativamente à validade e possibilidade de replicação da investigação em diferentes contextos.

Seguindo esta abordagem quantitativa aplicada ao plano da investigação social no desporto, a análise da interação entre membros de uma equipa a partir da teoria de grafos evidencia oportunidades únicas que, apesar de evidentes, assim são escassas na aplicação específica ao contexto desportivo. De facto, a extensão da teoria de grafos é muito mais evidente em contextos de trabalho colaborativo empresarial ou em ciências biológicas. No entanto, ao longo deste ensaio procurou-se evidenciar que esta abordagem é totalmente possível e viável no desporto, pelo que, a partir das diferentes técnicas e métricas associadas à teoria de grafos será possível aumentar o conhecimento e a validação científica sobre as formas de interação social que emergem no grupo de trabalho.

Desde a possibilidade de identificar as propriedades da rede e analisar a sua evolução ao longo da época de trabalho até à capacidade de associar as propriedades da rede com o desempenho coletivo da equipa, as possibilidades são múltiplas e de evidente utilidade. Adicionalmente, a capacidade de através de métodos matemáticos determinar a centralidade e o contributo individual de cada membro da equipa para o desenvolvimento do grupo é uma oportunidade única que valoriza a análise quantitativa, aproximando-a do que o processo observacional poderá determinar. Por fim, as métricas que identificam tendências de dependência no relacionamento poderá ser outro fator determinante para perceber quais os membros fundamentais que fazem a equipa evoluir na sua dinâmica corporativa.

Evidentemente que ao longo deste ensaio nenhuma aplicação prática foi testada. Para além disso, as métricas foram apresentadas de forma matemática e sem recurso a processos acessíveis de processamento de dados. No entanto, atualmente, existem múltiplas possibilidades no mercado que permitem computar os dados processando-os de forma Conexões: revista da Faculdade de Educação Física da UNICAMP, Campinas, v. 13, n. 3, p. 175-194, jul./set. 2015. 
automática. Um desses softwares de livre acesso é o $\operatorname{SocNetV} V^{33}$ desenvolvido para análise do comportamento social coletivo que permite, através da inserção dos dados sob forma de matriz de adjacência, computar todo o conjunto de métricas apresentadas e discutidas neste ensaio. No fundo, as opções no mercado online são de fácil obtenção e permitem o alastramento desta opção metodológica a qualquer investigador que, através da teoria de grafos e Social Network Analysis, tenha como objetivo identificar o processo de interação de uma equipa desportiva.

\section{REFERÊNCIAS}

${ }^{1}$ LUSHER, D., ROBINS, G., KREMER, P. The application of social network analysis to team sports. Measurement in Physical Education and Exercise Science, v. 14, n. 4, p. 211-224, june 2010.

${ }^{2}$ CLEMENTE, F.M. et al. Using network metrics to investigate football team players , connections: a pilot study. Motriz, Rio Claro, v . 20, n. 3, p. 262-271, set. 2014.

${ }^{3}$ CLEMENTE, F.M. et al. Using network metrics in soccer: a macro-analysis. Journal of Human Kinetics, v. 45, p. 123-134, july 2015.

${ }^{4}$ LIGHT SHIELDS, D. L. et al. The relationship between leadership behaviors and group cohesion in team sports. Journal of Psychology, v. 131, n. 2, p. 196-210, ago. 1997.

${ }^{5}$ CARRON, A. V.; BRAY, S. R.; EYS, M. A. Team cohesion and team success in sport. Journal of Sports Sciences, v. 20, n. 2, p. 119-26, feb. 2002.

${ }^{6}$ LOUGHEAD, T. M.; HARDY, J. An examination of coach and peer leader behaviors in sport. Pshychology of Sport and Exercise, v. 6, n. 3, p. 303-312, mar. 2005.

${ }^{7}$ MORGAN, P. B. C., FLETCHER, D.; SARKAR, M. Defining and characterizing team resilience in elite sport. Pshychology of Sport and Exercise, v.14, n. 4, p. 549-59, apr. 2013.

Conexões: revista da Faculdade de Educação Física da UNICAMP, Campinas, v. 13, n. 3, p. 175-194, jul./set. 2015. 
${ }^{8}$ FRANSEN, K. et al. The myth of the team captain as principal leader: extending the athlete leadership classification within sport teams. Journal of Sports Sciences, v. 32, n. 14, p. 1389-1397, dec. 2014.

${ }^{9}$ ABBOTT, A. Of time and space: the contemporary relevance of the chicago school. Social Forces, v. 75, n. 4, p. 1149-82, dec. 1997.

${ }^{10}$ MORENO, J. L. Who shall survive? foundations of sociometry, group psychotherapy and socio-drama. Oxford: Beacon House, 1953.

${ }^{11}$ KNOKE, D.; YANG, S. Social network analysis. London: SAGE, 2008.

${ }^{12}$ BARNES, J. A.; HARARY, F. Graph theory in network analysis. Soc Networks, v. 5, p. 235-44, apr. 1983.

${ }^{13}$ PAVLOPOULOS, G. A. et al. Using graph theory to analyze biological networks. BioData Min., v. 4, n. 1, p. 10, mar. 2011.

${ }^{14}$ HORVATH, S. Weighted network analysis: Applications in Genomics and Systems biology. New York: Springer, 2011.

${ }^{15}$ HAGGARTY, S. J.; CLEMONS, P. A.; SCHREIBER, S. L. Chemical genomic profiling of biological networks using graph theory and combinations of small molecule perturbations. Journal of the American Chemical Society, v.125, n. 35, p.10543-10545, apr. 2003.

${ }^{16}$ BURT, R. S.; KILDUFF, M., TASSELLI, S. Social network analysis: foundations and frontiers on advantage. Annual Review of Psychology, v. 64, p. 527-547, july 2013.

${ }^{17}$ HAMMOND, D. K.; VANDERGHEYNST, P., GRIBONVAL, R. Wavelets on graphs via spectral graph theory. Applied and Computational Harmonic Analysis, v. 30, n. 2, p.129-150, june 2011. 
${ }^{18}$ PRELL, C.; HUBACEK, K.; REED, M. Stakeholder analysis and social network analysis in natural resource management. Society \& Natural Resources, v. 22, n. 6, p. 501-518, 2009.

${ }^{19}$ LEVI-STRAUSS, C. The elementary structures of kinship. Boston, Beacon, 1969.

${ }^{20}$ PARK, R. The concept of social distance. Journal of Applied Sociology, v. 8, p. 339344, 1924.

${ }^{21}$ GRUND, T. U. Network structure and team performance: the case of english premier league soccer teams. Social Networks, v. 34, n. 4, p. 682-690, sept. 2012.

${ }^{22}$ WELLMAN, B.; BERKOWITZ, S. D. Structural analysis in the social sciences 2 social structures: a network approach. Cambridge: Cambridge University, 1988.

${ }^{23}$ OTTE, E.; ROUSSEAU, R. Social network analysis: a powerful strategy, also for the information sciences. Journal of Information Science, v. 28, n. 6, p. 441-453, jun. 2002.

${ }^{24}$ WETHERELL, C.; PLAKANS, A.; WELMANN, B. Social network, kinship, and community in Eastern Europe. Journal of Interdisciplinary History, v. 24, p. 639-663, oct. 1994.

${ }^{25}$ TICHY, N. M.; TUSHMAN, M. L.; FOMBRUN, C. Social network analysis for organizations. Academy of Management Review, v. 4, n. 4, p. 507-519, 1979.

${ }^{26}$ WASSERMAN, S.; FAUST, K. Social network analysis: Methods and applications. New York: Cambridge University, 1994.

${ }^{27}$ SCOTT, J. Social network analaysis. London: SAGE, 2000.

${ }^{28}$ HANNEMAN, R. A.; RIDDLE, M. Introduction to social network methods. Riverside, University of California, 2005. 
${ }^{29}$ GRÉHAIGNE, J. F.; BOUTHIER, D.; DAVID, B. Dynamic-system analysis of opponent relationship in collective actions in football. Journal of Sports Sciences, v. 15, n. 2, p.137-149, feb. 1997.

${ }^{30}$ WATTS, D. J.; STROGATZ, S. H. Collective dynamics of "small-world" networks. Nature. v. 393, p. 440-442, may 1998.

${ }^{31}$ RAVASZ, E. et al Hierarchical organization of modularity in metabolic networks. Science, v. 297, n. 5586, p.1551-1555, nov. 2002.

${ }^{32}$ COUCEIRO, M. S.; CLEMENTE, F. M.; MARTINS, F. M. L. Toward the evaluation of research groups based on scientific co-authorship networks : the robocorp case study. The Arab Gulf Journal of Scientific Research, v. 31, n. 1, p. 36-52, dec. 2013.

${ }^{33}$ KALAMARAS, D. Social networks visualizer (SocNetV): social network analysis and visualization software. Social Networks Visualizer. Disponível em: http://socnetv.sourceforge.net, 2014.

Recebido em: 05 maio 2015 Aceito em: 24 ago. 2015 Contato: filipe.clemente@gmail.com 